\title{
ESPÉCIES POTENCIAIS PARA RECUPERAÇÃO DE ÁREAS DE FLORESTA ESTACIONAL SEMIDECIDUAL COM EXPLORAÇÃO DE MINÉRIO DE FERRO NA SERRA DO ESPINHAÇO
}

\author{
POTENTIAL SPECIES FOR RECOVERY AREAS SEMIDECIDUOUS FOREST IN \\ IRON EXPLORATION IN THE SERRA ESPINHAÇO
}

\author{
Milton Serpa de MEIRA JUNIOR'; Israel Marinho PEREIRA²; Evandro Luiz Mendonça \\ MACHADO' ${ }^{2}$; Silvia da Luz Lima MOTA ${ }^{3}$; Thiago José Ornelas OTONI ${ }^{4}$ \\ 1. Mestrando em Ciências Florestais no curso de pós-graduação da Universidade de Brasília, Brasília, DF, Brasil. \\ milton.serpa@gmail.com ; 2. Professor, Departamento de Engenharia Florestal, Universidade Federal dos Vales do Jequitinhonha e \\ Mucuri, Diamantina, MG, Brasil; 3. Doutoranda em Ciências Florestais na Universidade de Brasília, Brasília, DF, Brasil; 4. Mestre em \\ Ciências Florestais na Universidade Federal dos Vales do Jequitinhonha e Mucuri, Diamantina, MG, Brasil.
}

RESUMO: O objetivo deste trabalho foi avaliar a flora arbustiva-arbórea em um fragmento de floresta estacional semidecidual na região da Serra do Espinhaço para propor espécies para recuperação das áreas degradadas pela exploração de minério de ferro em ecossistemas congêneres. O levantamento florístico e estrutural da vegetação arbustivoarbórea foi realizado em setembro de 2007 por meio do método dos pontos quadrantes, tendo como critério de inclusão altura do fuste superior a 1,3 m. Os indivíduos foram estratificados entre adulto (DAP $\geq 5,0 \mathrm{~cm}$ ) e juvenil (DAP $<5,0 \mathrm{~cm}$ ). Foram amostrados 90 pontos, onde foram identificadas 94 espécies, 70 gêneros e 32 famílias botânicas. Para o índice de diversidade de Shannon, os valores apresentados para cada compartimento foram 3,96 nat.ind ${ }^{-1}$ e 3,94 nat.ind ${ }^{-1}$; e a equabilidade de Pielou foi de 0,896 e 0,938, respectivamente, para os estrados juvenil e adulto. As famílias que mais se destacaram quanto ao número de espécies foram Myrtacea (17), Fabaceae (12), Rubiaceae (8), Euphrobiaceae (6), Sapindaceae (5) e Lauraceae (5). Para a recuperação da área foram selecionados sete grupos de espécies que serão utilizados. As características das espécies foram suficientes para a definição de grupos funcionais que poderão ser utilizados na recuperação de áreas degradadas.

PALAVRAS-CHAVE: Restauração ambiental. Modelos de plantio. Grupos funcionais e sucessão ecológica.

\section{INTRODUÇÃO}

A Cadeia do Espinhaço é a segunda mais longa formação de montanhas do Brasil e se estende por mais de mil quilômetros na direção norte-sul, apresentando seu limite norte na serra da Jacobina BA, e sul na serra do Ouro Branco - MG. A Cadeia do Espinhaço apresenta dois blocos devido ao seu estreitamento no norte do estado de Minas Gerais, sendo a porção norte no estado da Bahia, conhecida como Chapada Diamantina, e a porção sul no estado de Minas Gerais, conhecida como Serra do Espinhaço.

Sua extensão longitudinal varia aproximadamente entre 50 e $100 \mathrm{~km}$ e constitui um divisor de águas entre a bacia hidrográfica do rio São Francisco, a oeste, e as bacias dos rios que drenam para o Atlântico, a leste (VIEIRA et al., 2005). Essa Cadeia foi considerada pela Unesco em 2005 a sétima reserva da biosfera brasileira, devido a sua enorme biodiversidade de recursos naturais.

O mosaico de fitofisionomias é encontrado de norte a sul na Cadeia do Espinhaço e a paisagem e a composição da flora sofrem forte influência de três grandes Biomas brasileiros: a Mata Atlântica, mais ao sul da cadeia; o Cerrado na porção mais central e a Caatinga mais ao norte da mesma (HARLEY, 1995). Dentre as formações vegetais de Minas Gerais inseridas na Serra do Espinhaço estão as florestas estacionais semideciduais (FES), caracterizadas pela sazonalidade climática que determina a perda foliar (20 a $50 \%$ de deciduidade) dos indivíduos arbóreos e arbustivos, em resposta à deficiência hídrica ou a queda de temperatura nos meses mais frios e secos (VELOSO et al.,1991).

No estado de Minas Gerais, as fisionomias florestais se estendiam por uma vasta região do centro-sul e leste do estado (IBGE, 1993), porém, o crescimento da população humana tem acelerado a remoção da cobertura vegetal, ao aumentar a demanda por produtos florestais e terras para agricultura, pecuária, abertura de estradas, centros urbanos (MYERS et al., 2000) e exploração mineral.

Embora a maioria dos solos da Cadeia do Espinhaço sejam provenientes de quartizitos e arenitos, existem, principalmente na porção mineira da Cadeia, áreas sobre solos ricos em minério de ferro (GONTIJO, 2008), que têm atraído grandes empresas mineradoras.

Depois da extração do minério de ferro, o substrato fica endurecido e empobrecido, dificultando os processos de regeneração natural ou 
mesmo de revegetação artificial (TEIXEIRA; LEMOS-FILHO, 2002). E o processo de revegetação requer o emprego de técnicas adequadas, geralmente, definidas em função de avaliações detalhadas das condições locais e da utilização dos conhecimentos científicos existentes (PEREIRA et al., 2010). O conhecimento e utilização de informações ecológicas e silviculturais, tais como exigência de luz, relações planta-animais, freqüência e densidade natural, podem levar ao conhecimento da combinação ideal de espécies dentro dos mosaicos de estádios sucessionais, semelhantes aos formados nas florestas tropicais naturais (PEREIRA et al., 2010).

O objetivo deste trabalho foi avaliar a flora arbustiva-arbórea em um fragmento de floresta estacional semidecidual a fim de agrupar as espécies pelas características ecológicas, de forma a subsidiar futuras ações voltadas à recuperação de áreas degradadas pela exploração de mineiro de ferro em ecossistemas congêneres.

\section{MATERIAL E MÉTODOS}

\section{Caracterização e localização da área}

A área de estudo pertence ao domínio geomorfológico da Serra do Espinhaço, entre as Bacias do Rio Jequitinhonha e do Rio Doce e localizada no município de Conceição do Mato Dentro, MG. Nessa região encontram-se áreas com solos ricos em minério de ferro, onde tem-se constatado o estabelecimento de grandes empresas empreendimentos de extração desse mineral.

A área inventariada apresenta formação florestal característica de Floresta Estacional Semidecidual Montana, segundo classificação do IBGE (VELOSO et al., 1991). O fragmento possui uma área de 22 ha contínuos de mata $(670864,46 \mathrm{~m}$ a $670546,80 \mathrm{~m} \mathrm{~S}$ e $7948340,52 \mathrm{~m}$ a $7948427,28 \mathrm{~m}$ W). A região possui regime climático marcado por verão quente e úmido com chuvas concentradas e inverno frio com mais de três meses de estiagem por ano (Cwa de Köppen: mesotérmico úmido subtropical) (LANA et al., 2001).

\section{Levantamento florístico e fitossociológico}

O levantamento florístico e fitossociológico da vegetação arbustivo-arbórea foi realizado em setembro de 2007, por meio do método dos pontos quadrantes (COTTAM; CURTIS, 1956) de acordo com o método das distâncias mínimas, proposto por Martins (1979). Neste trabalho, foram amostrados 90 pontos distribuídos sistematicamente a cada 20 metros, devido a alta densidade de indivíduos na área. Ao alcançar cada borda do fragmento iniciava- se uma nova linha com 50 metros de distância ortogonal em relação ao último ponto. $\mathrm{O}$ caminhamento foi realizado de forma a amostrar o maior número de pontos possíveis na área, sendo eles orientados com o auxílio de uma cruzeta de metal, balizas e uma bússola.

Em cada quadrante foi amostrado o indivíduo arbustivo-arbóreo vivo que apresentou a menor distância ao centro do ponto que atendesse o critério de inclusão adotado neste trabalho que foi todos os indivíduos que apresentassem fuste com altura superior a 1,3 metros em relação ao solo, ou seja, que apresentassem fuste com altura superior a 1,3 metros, ou seja, apresentassem $D A P$ (diâmetro a 1,3 metros do solo).

A área amostral foi definida a partir das métricas clássicas utilizadas no método de quadrantes descritas nas equações seguintes (COTTAM; CURTIS, 1956). Essa área é variável, diretamente dependente da distância média árvoreponto e relaciona-se com o padrão estrutural da floresta. Para se definir a distância média $\left(\mathrm{d}_{\mathrm{m}}\right)$ árvore-ponto (Expressão 2), é necessário fazer a correção das distâncias individuais medidas em campo. Por definição do método, a distância corrigida $\left(\mathrm{d}_{\mathrm{c}}\right)$ é equivalente à distância individual $(\mathrm{d}$, a distância árvore-ponto medida durante o levantamento de campo) somada ao respectivo raio (na altura de 1,3 $\mathrm{m}$ do solo) do indivíduo amostrado, como indicado na Expressão 1:

$d_{c}=d+(D A P / 2)$

Em que: $\mathbf{d}_{\mathbf{c}}$ representa o valor da distância corrigida árvore-ponto, em metro; d é distância entre o centro do ponto e o perímetro do fuste, em metro; DAP é o valor do diâmetro em metro, medido à $1,3 \mathrm{~m}$ do nível do solo para o respectivo indivíduo amostrado.

$d_{m}=\Sigma_{1}^{n} d_{e} / n$

Em que: $\mathbf{d}_{\mathbf{m}}$ representa o valor da distância média árvore-ponto, em metro; dc é a distância corrigida, em metro, de cada indivíduo; $n$ é o número de indivíduos amostrados.

Para o cálculo da área amostrada, considerase que cada indivíduo ocupa uma área quadrada com dimensões dependentes do valor de $d_{m}$ (Expressão 1) como dado pela expressão abaixo:

$A_{e q}=\left(d_{m}^{2} \times n\right) / 10.000^{2}$

Em que: $\mathbf{A}_{\text {eq }}$ é o valor da área amostral equivalente, em hectare; $\mathbf{d}_{\mathbf{m}}$ é a distância média árvore-ponto, em metro; $\mathbf{n}$ é o número de indivíduos amostrados. 
O material botânico foi coletado e identificado por meio de consultas à literatura e especialistas ou por comparações com espécimes existentes nos Herbário da UFVJM, UFMG e ESAL. O sistema de classificação utilizado foi o APG III (ANGIOSPERM PHYLOGENY GROUP, 2009).

As espécies amostradas foram classificadas quanto às guildas de dispersão e regeneração. As informações das espécies para a classificação quanto à guilda de dispersão foram coletadas na literatura de acordo com Van der Pijl (1982), como: anemocóricas (ANE), zoocóricas (ZOO), autocóricas (AUTO). Quanto à classificação das espécies em guildas de regeneração, adotou-se a definição proposta por Gandolfi et al. (1995), pioneiras $(\mathrm{P})$, secundárias iniciais $(\mathrm{SI})$ e secundárias tardias (ST). Para a obtenção de informações ecológicas sobre as espécies, foram utilizadas informações encontradas na literatura (MEIRANETO; MARTINS, 2002; SILVA, 2002; SOUZA et al., 2002; DURIGAN et al., 2004; RESSEL et al., 2004; PAULA et al., 2004; TAKAHASHI; FIANA, 2004; CATHARINO et al., 2006; PEREIRA et al., 2006; YAMAMOTO et al., 2007; LOPES et al., 2008; DIAS-NETO et al., 2009) e observações em campo. As espécies foram classificadas quanto ao ritmo de crescimento de acordo com informações contidas na literatura (CARVALHO, 2003, 2006, 2008; DURIGAN et al.; 2004).

Os grupos de espécies foram determinados com base nas informações obtidas na matriz de guildas ecológica das espécies, adicionadas ao grupo ecológico que cada espécie pertence como proposto por Pereira (2006), em que: Grupo I: pioneira atrativa à fauna (dispersão zoocórica), Grupo II: pioneira ativadora da sucessão (ritmo de crescimento rápido), Grupo III: pioneira atrativa a fauna e ativadora da sucessão, Grupo IV: secundária inicial atrativa à fauna, Grupo V: secundária inicial ativadora da sucessão, Grupo VI: secundária inicial atrativa à fauna e ativadora da sucessão e Grupo VII: secundária tardia.

Os indivíduos registrados foram categorizados em dois compartimentos de desenvolvimento, sendo considerados adultos todos os indivíduos com DAP $\geq 5,0 \mathrm{~cm}$ e altura do fuste superior a $1,30 \mathrm{~m}$ e juvenil, indivíduos com $\mathrm{DAP}<5,0 \mathrm{~cm}$ e altura de fuste superior a $1,30 \mathrm{~m}$, de forma que fossem amostrados nos compartimentos adulto e regenerante as espécies de maneira quantitativa e qualitativa.

A diversidade foi expressa pelo índice de diversidade de Simpson (1/D), Shannon e equabilidade de Pielou (J') (ZAR, 1996). Para verificar a distribuição de frequência de indivíduos arbustivo-arbóreos nas guildas de regeneração e dispersão eram independentes em cada compartimento, juvenil e adulto, foi aplicado o teste de Qui-quadrado para tabela de contingência (ZAR, 1996).

\section{RESULTADOS}

Foram amostrados 360 indivíduos distribuídos em 94 espécies identificadas, 70 gêneros e 32 famílias botânicas (Tabela 1). Não foi possível identificar 17 morfoespécies amostradas que foram morfologicamente agrupadas para os cálculos de estrutura. Esses indivíduos não identificados foram retirados da análise florística. $\mathrm{O}$ compartimento adulto foi representado por 67 espécies e o compartimento juvenil apresentou 83 espécies.

Para o índice de diversidade de Shannon, os valores apresentados para cada compartimento foram 3,96 nat.ind ${ }^{-1}$ (juvenil) e 3,94 nat.ind $^{-1}$ (adulto) e para o índice de equabilidade de Pielou foi de 0,896 para o compartimento juvenil e 0,938 para o adulto.

Tabela 1. Lista florística de uma área de Floresta Estacional Semidecidual, em Conceição do Mato Dentro MG. Sendo, $\mathrm{P}=$ pioneira, $\mathrm{SI}=$ secundárias iniciais, $\mathrm{ST}=$ secundárias tardias, $\mathrm{ZOO}=$ zoocóricas, $\mathrm{ANE}=$ anemocóricas; $\mathrm{AUT}=$ autocóricas; $\mathrm{R}=$ guilda de regeneração; $\mathrm{D}=$ guilda de dispersão; $\mathrm{Ju}=$ juvenil; $\mathrm{Ad}=$ adulto; $\mathrm{RC}=$ ritmo de crescimento; $\mathrm{R}=$ rápido; $\mathrm{M}=$ médio; $\mathrm{L}=$ lento

\begin{tabular}{lccccc}
\hline FAMÍLIA & \multirow{2}{*}{ RRC } & \multirow{2}{*}{$\mathrm{R}$} & \multicolumn{3}{c}{ NI } \\
Espécie & & & & & \\
\hline ANACARDIACEAE & $\mathrm{L}$ & $\mathrm{ST}$ & $\mathrm{ANE}$ & 1 & 1 \\
Astronium concinnum Schott ex Spreng. & $\mathrm{L}$ & $\mathrm{P}$ & $\mathrm{ANE}$ & 2 & 1 \\
Myracrodruon urundeuva Allemão & $\mathrm{R}$ & $\mathrm{SI}$ & ZOO & 1 & 1 \\
Tapirira guianensis Aubl. & $\mathrm{R}$ & $\mathrm{SI}$ & ZOO & 0 & 1 \\
Tapirira obtusa (Benth.) J.D.Mitch. & & & & & \\
ANNONACEAE & $\mathrm{R}$ & SI & ZOO & 3 & 1 \\
Annona acutiflora Mart. & & & &
\end{tabular}




\begin{tabular}{|c|c|c|c|c|c|}
\hline $\begin{array}{l}\text { FAMÍLIA } \\
\text { Espécie }\end{array}$ & $\mathbf{R R C}$ & $\mathrm{R}$ & $\mathrm{D}$ & $J^{\mathrm{P}}$ & Ad \\
\hline Guatteria sellowiana Schltdl. & $\mathrm{M}$ & SI & $\mathrm{ZOO}$ & 1 & 0 \\
\hline Xylopia brasiliensis Spreng. & $\mathrm{R}$ & ST & $\mathrm{ZOO}$ & 10 & 1 \\
\hline Xylopia sericea A.St.-Hil. & $\mathrm{R}$ & ST & $\mathrm{ZOO}$ & 14 & 4 \\
\hline \multicolumn{6}{|l|}{ APOCYNACEAE } \\
\hline Aspidosperma cylindrocarpon Müll.Arg. & $\mathrm{L}$ & SI & ANE & 1 & 1 \\
\hline Aspidosperma polyneuron Müll.Arg. & M & ST & ANE & 0 & 1 \\
\hline \multicolumn{6}{|l|}{ AQUIFOLIACEAE } \\
\hline Ilex affinis Gardn. & $\mathrm{L}$ & SI & $\mathrm{ZOO}$ & 1 & 1 \\
\hline $\begin{array}{l}\text { Ilex theezans Mart. ex Reissek } \\
\text { ASTERACEAE }\end{array}$ & $\mathrm{R}$ & SI & $\mathrm{ZOO}$ & 1 & 0 \\
\hline Eremanthus erythropappus (DC.) MacLeish & $\mathrm{R}$ & $\mathrm{P}$ & ANE & 0 & 3 \\
\hline \multicolumn{6}{|l|}{ BIGNONIACEAE } \\
\hline Cybistax antisyphilitica (Mart.) Mart. & M & SI & ANE & 3 & 0 \\
\hline \multicolumn{6}{|l|}{ BORAGINACEAE } \\
\hline $\begin{array}{l}\text { Cordia ecalyculata Vell. } \\
\text { BURSERACEAE }\end{array}$ & M & SI & $\mathrm{ZOO}$ & 4 & 1 \\
\hline Protium heptaphyllum (Aubl.) Marchand & M & $\mathrm{P}$ & $\mathrm{ZOO}$ & 5 & 3 \\
\hline \multicolumn{6}{|l|}{ CELASTRACEAE } \\
\hline $\begin{array}{l}\text { Maytenus boaria Molina } \\
\text { CLUSIACEAE }\end{array}$ & $\mathrm{L}$ & ST & $\mathrm{ZOO}$ & 1 & 0 \\
\hline Garcinia gardneriana (Planch. \& Triana) Zappi & M & SI & $\mathrm{ZOO}$ & 2 & 0 \\
\hline $\begin{array}{l}\text { Kielmeyera lathrophyton Saddi } \\
\text { EUPHORBIACEAE }\end{array}$ & $\mathrm{L}$ & $\mathrm{P}$ & ANE & 1 & 0 \\
\hline $\begin{array}{l}\text { Alchornea triplinervia (Sprengel) Müll.Arg. } \\
\text { EUPHORBIACEAE }\end{array}$ & $\mathrm{R}$ & SI & $\mathrm{ZOO}$ & 2 & 0 \\
\hline Croton piptocalyx Müll.Arg. & $\mathrm{R}$ & $\mathrm{P}$ & AUT & 2 & 0 \\
\hline Mabea fistulifera Mart. & $\mathrm{R}$ & $\mathrm{P}$ & ANE & 5 & 3 \\
\hline Manihot brachyloba Müll.Arg. & $\mathrm{R}$ & $\mathrm{P}$ & AUT & 0 & 1 \\
\hline $\begin{array}{l}\text { Maprounea guianensis Aubl. } \\
\text { FABACEAE }\end{array}$ & M & SI & AUT & 2 & 0 \\
\hline Bauhinia longifolia (Bong.) D.Dietr. & M & $\mathrm{P}$ & AUT & 0 & 3 \\
\hline Copaifera langsdorffii Desf. & M & SI & $\mathrm{ZOO}$ & 5 & 7 \\
\hline Tachigali rugosa (Mart. ex Benth.) Zarucchi e Pipoly & M & SI & ANE & 1 & 5 \\
\hline Dalbergia nigra (Vell.) Allemão ex Benth. & M & SI & ANE & 0 & 8 \\
\hline Dalbergia villosa (Benth.) Benth. & $\mathrm{L}$ & SI & ANE & 1 & 1 \\
\hline Diplotropis ferruginea Benth. & $\mathrm{L}$ & $\mathrm{P}$ & AUT & 0 & 1 \\
\hline Hymenolobium heringerianum Rizzini & M & SI & ANE & 1 & 0 \\
\hline Machaerium nyctitans (Vell.) Benth. & $\mathrm{L}$ & SI & ANE & 0 & 3 \\
\hline Machaerium stipitatum (DC.) Vogel & $\mathrm{M}$ & SI & ANE & 2 & 0 \\
\hline Machaerium villosum Vogel & $\mathrm{R}$ & SI & ANE & 4 & 2 \\
\hline Swartzia myrtifolia J.E.Sm. & $\mathrm{SC}$ & SI & $\mathrm{ZOO}$ & 1 & 0 \\
\hline $\begin{array}{l}\text { Piptadenia gonoacantha (Mart.) J.F.Macbr. } \\
\text { HYPERICACEAE }\end{array}$ & $\mathrm{R}$ & $\mathrm{P}$ & AUT & 0 & 1 \\
\hline Vismia brasiliensis Choisy & $\mathrm{M}$ & SI & $\mathrm{ZOO}$ & 3 & 1 \\
\hline
\end{tabular}




\begin{tabular}{|c|c|c|c|c|c|}
\hline $\begin{array}{l}\text { FAMÍLIA } \\
\text { Espécie }\end{array}$ & RRC & $\mathrm{R}$ & $\mathrm{D}$ & & I \\
\hline \multicolumn{6}{|l|}{ LAMIACEAE } \\
\hline Hyptidendron asperrimum (Epling) Harley & M & SI & $\mathrm{ZOO}$ & 2 & 3 \\
\hline \multicolumn{6}{|l|}{ LAURACEAE } \\
\hline Aiouea saligna Meisn. & $\mathrm{SC}$ & $\mathrm{P}$ & $\mathrm{ZOO}$ & 2 & 0 \\
\hline Cinnamomum erythropus (Nees e Mart) Kosterm. & $\mathrm{L}$ & SI & $\mathrm{ZOO}$ & 0 & 1 \\
\hline Nectandra cissiflora Nees & M & ST & $\mathrm{ZOO}$ & 9 & 4 \\
\hline Nectandra lanceolata Nees & M & ST & $\mathrm{ZOO}$ & 0 & 2 \\
\hline Ocotea pulchella Mart. & M & SI & $\mathrm{ZOO}$ & 0 & 1 \\
\hline \multicolumn{6}{|l|}{ MALPIGHIACEAE } \\
\hline Byrsonima crassifolia (L.) Kunth & $\mathrm{L}$ & SI & $\mathrm{ZOO}$ & 1 & 2 \\
\hline \multicolumn{6}{|l|}{ MELASTOMATACEAE } \\
\hline Miconia brunnea Mart. & $\mathrm{L}$ & ST & $\mathrm{ZOO}$ & 1 & 0 \\
\hline $\begin{array}{l}\text { Miconia cinnamomifolia (DC.) Naudin } \\
\text { MELIACEAE }\end{array}$ & M & $\mathrm{P}$ & $\mathrm{ZOO}$ & 1 & 2 \\
\hline Cabralea canjerana (Vell.) Mart. & $\mathrm{M}$ & ST & $\mathrm{ZOO}$ & 3 & 4 \\
\hline Trichilia hirta $\mathrm{L}$. & $\mathrm{M}$ & SI & $\mathrm{ZOO}$ & 0 & 1 \\
\hline \multicolumn{6}{|l|}{ MORACEAE } \\
\hline Ficus gomelleira Kunth e Bouché & M & SI & $\mathrm{ZOO}$ & 1 & 0 \\
\hline $\begin{array}{l}\text { Sorocea bonplandii (Baill.) W.Burger } \\
\text { MYRSINACEAE }\end{array}$ & $\mathrm{L}$ & SI & $\mathrm{ZOO}$ & 2 & 0 \\
\hline Myrsine gardneriana A.DC. & M & SI & $\mathrm{ZOO}$ & 0 & 1 \\
\hline $\begin{array}{l}\text { Myrsine guianensis (Aubl.) Kuntze } \\
\text { MYRTACEAE }\end{array}$ & $\mathrm{R}$ & $\mathrm{P}$ & $\mathrm{ZOO}$ & 1 & 0 \\
\hline Blepharocalyx salicifolius (Kunth) O.Berg & $\mathrm{L}$ & SI & $\mathrm{ZOO}$ & 1 & 1 \\
\hline Calyptranthes clusiifolia O.Berg & $\mathrm{L}$ & SI & $\mathrm{ZOO}$ & 1 & 0 \\
\hline Calyptranthes concinna DC. & $\mathrm{L}$ & $\mathrm{P}$ & $\mathrm{ZOO}$ & 8 & 2 \\
\hline Calyptranthes lucida Mart. ex DC. & $\mathrm{L}$ & ST & $\mathrm{ZOO}$ & 1 & 0 \\
\hline Eugenia acutata Miq. & M & ST & $\mathrm{ZOO}$ & 1 & 1 \\
\hline Eugenia brasiliensis Lam. & $\mathrm{L}$ & ST & $\mathrm{ZOO}$ & 1 & 0 \\
\hline Eugenia florida DC. & $\mathrm{L}$ & ST & $\mathrm{ZOO}$ & 7 & 2 \\
\hline Eugenia francavilleana O.Berg & $\mathrm{L}$ & ST & $\mathrm{ZOO}$ & 4 & 3 \\
\hline Eugenia involucrata DC. & $\mathrm{L}$ & SI & $\mathrm{ZOO}$ & 3 & 3 \\
\hline Eugenia pyriformis Cambess. & $\mathrm{L}$ & ST & $\mathrm{ZOO}$ & 2 & 2 \\
\hline Myrcia retorta Cambess. & $\mathrm{L}$ & $\mathrm{P}$ & $\mathrm{ZOO}$ & 3 & 1 \\
\hline Myrcia pulchra Kiaersk. & $\mathrm{R}$ & ST & $\mathrm{ZOO}$ & 1 & 0 \\
\hline Myrcia splendens (Sw.) DC. & $\mathrm{L}$ & SI & $\mathrm{ZOO}$ & 8 & 12 \\
\hline Pimenta pseudocaryophyllus (Gomes) Landrum & $\mathrm{R}$ & $\mathrm{P}$ & $\mathrm{ZOO}$ & 0 & 1 \\
\hline Plinia grandifolia (Mattos) Sobral & $\mathrm{L}$ & SI & $\mathrm{ZOO}$ & 4 & 1 \\
\hline $\begin{array}{l}\text { Siphoneugena densiflora O.Berg } \\
\text { RUBIACEAE }\end{array}$ & $\mathrm{L}$ & ST & $\mathrm{ZOO}$ & 2 & 1 \\
\hline Alibertia edulis (L.C.Rich.) A.Rich. ex DC. & $\mathrm{M}$ & ST & $\mathrm{ZOO}$ & 2 & 1 \\
\hline Amaioua guianensis Aubl. & $\mathrm{SC}$ & ST & $\mathrm{ZOO}$ & 6 & 1 \\
\hline Chomelia brasiliana A.Rich. & $\mathrm{L}$ & ST & $\mathrm{ZOO}$ & 1 & 0 \\
\hline
\end{tabular}




\begin{tabular}{|c|c|c|c|c|c|}
\hline $\begin{array}{l}\text { FAMÍLIA } \\
\text { Espécie }\end{array}$ & $\mathbf{R R C}$ & $\mathrm{R}$ & $\mathrm{D}$ & $\begin{aligned} 1 \\
J u\end{aligned}$ & $A d$ \\
\hline Cordiera sessilis (Vell.) Kuntze & $\mathrm{L}$ & $\mathrm{P}$ & $\mathrm{ZOO}$ & 2 & 0 \\
\hline Coussarea hydrangeifolia (Benth.) Benth. e Hook.f. ex Müll.Arg. & M & $\mathrm{P}$ & $\mathrm{ZOO}$ & 1 & 0 \\
\hline Coutarea hexandra (Jacq.) K.Schum. & M & SI & ANE & 0 & 1 \\
\hline Psychotria vellosiana Benth. & M & SI & $\mathrm{ZOO}$ & 1 & 1 \\
\hline $\begin{array}{l}\text { Rudgea gardenioides (Cham.) Müll.Arg. } \\
\text { RUTACEAE }\end{array}$ & $\mathrm{SC}$ & ST & $\mathrm{ZOO}$ & 1 & 0 \\
\hline Esenbeckia febrifuga (A.St.-Hil.) A.Juss. ex Mart. & $\mathrm{L}$ & SI & AUT & 1 & 0 \\
\hline $\begin{array}{l}\text { Hortia brasiliana Vand. ex DC. } \\
\text { SALICACEAE }\end{array}$ & $\mathrm{SC}$ & ST & $\mathrm{ZOO}$ & 1 & 1 \\
\hline $\begin{array}{l}\text { Casearia arborea (L.C.Rich.) Urb. } \\
\text { SAPINDACEAE }\end{array}$ & M & SI & $\mathrm{ZOO}$ & 2 & 2 \\
\hline $\begin{array}{l}\text { Cupania vernalis Cambess. } \\
\text { Diatenopteryx sorbifolia } \text { Radlk. } \\
\text { Dilodendron bipinnatum Radlk. } \\
\text { Matayba elaeagnoides Radlk. }\end{array}$ & $\begin{array}{l}\mathrm{M} \\
\mathrm{SC} \\
\mathrm{L} \\
\mathrm{M}\end{array}$ & $\begin{array}{l}\text { SI } \\
\mathrm{P} \\
\mathrm{P} \\
\mathrm{P}\end{array}$ & $\begin{array}{l}\text { ZOO } \\
\text { ANE } \\
\text { ZOO } \\
\text { ZOO }\end{array}$ & $\begin{array}{l}2 \\
3 \\
3 \\
1\end{array}$ & $\begin{array}{l}0 \\
1 \\
0 \\
1\end{array}$ \\
\hline SAPINDACEAE & & & & & \\
\hline $\begin{array}{l}\text { Toulicia laevigata Radlk. } \\
\text { SAPOTACEAE }\end{array}$ & M & SI & ANE & 8 & 1 \\
\hline Pouteria ambelaniifolia (Sandwith) T.D.Penn. & $\mathrm{SC}$ & ST & $\mathrm{ZOO}$ & 0 & 1 \\
\hline Pouteria caimito (Ruiz e Pav.) Radlk. & $\mathrm{M}$ & SI & $\mathrm{ZOO}$ & 0 & 2 \\
\hline Picrasma crenata (Vell.) Eichler & $\mathrm{L}$ & SI & $\mathrm{ZOO}$ & 0 & 1 \\
\hline SIPARUNACEAE & & & & & \\
\hline Siparuna guianensis Aubl. & $\mathrm{R}$ & SI & ANE & 26 & 4 \\
\hline VERBENACEAE & & & & & \\
\hline Aloysia virgata (Ruiz e Pav.) A.Juss. & $\mathrm{R}$ & $P$ & ANE & 2 & 0 \\
\hline Citharexylum myrianthum Cham. & $\mathrm{SC}$ & SI & $\mathrm{ZOO}$ & 1 & 0 \\
\hline
\end{tabular}

Dentre as famílias encontradas nos dois compartimentos algumas se destacaram quanto à riqueza de espécies: Myrtacea (17), Fabaceae (12), Rubiaceae (8), Euphorbiaceae (6), Sapindaceae (5) e Lauraceae (5), as quais juntas representam 57\% das espécies identificadas.

No compartimento arbustivo-arbóreo formado por indivíduos adultos (DAP $\geq 5,0 \mathrm{~cm}$ ), foram estimados 1.813 indivíduos por hectare (Tabela 1). As maiores classes de DAP foram representadas principalmente por $D$. ferruginea, que pertence ao grupo das pioneiras e T. rugosa, $A$. cylindrocarpon, B. coriacea, C. canjerana, espécies climácicas.

No compartimento arbustivo-arbóreo formado por indivíduos jovens (DAP $<5 \mathrm{~cm}$ ) foram encontrados 74 espécies e 3.059 indivíduos por hectare, com DAP médio de $2,67 \mathrm{~cm}$ e altura média de $4,30 \mathrm{~m}$.

Para as espécies pioneiras, os indivíduos dos compartimentos adulto e juvenil apresentaram um número de indivíduos que atende o esperado, já as espécies secundárias iniciais apresentaram uma frequência maior que a esperada, e as espécies secundárias tardias apresentaram uma frequência menor que o esperado pelo teste Qui-quadrado (Tabela 2).

Em relação às guildas de dispersão todas apresentaram um número de indivíduos que atende ao esperado para os dois compartimentos (Tabela 3), não havendo diferença significativa entre os compartimentos para todos os tipos de dispersão.

A relação das 94 espécies registradas nos levantamentos florísticos e fitossociológicos em relação aos sete grupos de aptidões ecológicas definidos neste trabalho encontra-se na Tabela 4. Verifica-se que 11 espécies foram classificadas como pioneiras atrativas à fauna (grupo I), oito como pioneiras ativadoras da sucessão (grupo II), três espécies foram classificadas como pioneiras atrativas à fauna e ativadoras da sucessão (grupo III), trinta espécies como clímax exigente de luz atrativas à fauna (grupo IV), nove como clímax exigente de luz ativadoras da sucessão, seis como 
clímax exigente de luz atrativas à fauna e ativadoras da sucessão (grupo VI) e outras 23 espécies como clímax tolerante à sombra (grupo VII).

Tabela 2. Comparação do número de espécies dos compartimentos juvenil e adulto em relação às guildas de regeneração para dados de vegetação arbustiva-arbórea em uma floresta estacional semidecidual em Conceição do Mato Dentro - MG. Em que, SI: secundárias iniciais, ST: secundárias tardias e P: Pioneiras. Entre parênteses estão os valores esperados

\begin{tabular}{cccccc}
\hline Habitat & Adulto & Juvenil & Total & $X^{2}$ & $P$ \\
\hline SI & $75(66,57)$ & $102(110,43)$ & 177 & 0,95 & 0,19 \\
ST & $30(37,23)$ & $69(61,77)$ & 99 & 1,22 & 0,13 \\
P & $24(25,2)$ & $43(41,8)$ & 67 & 0,02 & 0,76 \\
\hline Total & 129 & 214 & 343 & & \\
\hline$X^{2}$ & 2,18 & 1,32 & & & \\
$P$ & 0,28 & 0,47 & & & \\
\hline
\end{tabular}

Tabela 3. Comparação do número de espécies dos compartimentos juvenil e adulto em relação às guildas de dispersão para dados de vegetação arbustiva-arbórea em uma floresta estacional semidecidual em Conceição do Mato Dentro - MG.

\begin{tabular}{cccccc}
\hline Dispersão & Adulto & Juvenil & Total & $\mathrm{X} 2=$ & $\mathrm{P}<$ \\
\hline Anemocóricas & $16(14,24)$ & $15(16,76)$ & 100 & 0,207 & 0,525 \\
Autocóricas & $4(3,21)$ & $3(3,79)$ & 100 & 0,047 & 0,551 \\
Zoocóricas & $42(44,55)$ & $55(52,45)$ & 100 & 0,174 & 0,604 \\
\hline Total & 62 & 73 & 400 & & \\
$\mathrm{X} 2=$ & 38,296 & 2,253 & & & \\
$\mathrm{P}<$ & 0,757 & 0,790 & & & \\
\hline
\end{tabular}

Tabela 4. Matriz com as 90 espécies arbustivo-arbóreas, registradas nos levantamentos florístico e fitossociológico em Conceição do Mato Dentro, MG, distribuídas nas categorias de função ecológica das espécies. Em que: presença (x) e ausência (-). Atrativa à fauna (DZ = espécie com dispersão zoocórica). Espécies ativadoras da sucessão ecológica $(\mathrm{CR}=$ espécie de crescimento rápido; $\mathrm{FBN}=$ espécies com fixação biológica de nitrogênio). Grupos ( $\mathrm{I}=$ pioneira atrativa à fauna; $\mathrm{II}=$ pioneira ativadora da sucessão; $\mathrm{III}=$ pioneira atrativa à fauna e ativadora da sucessão; $\mathrm{IV}=$ secundária inicial atrativa à fauna; $\mathrm{V}=$ secundária inicial ativadora da sucessão; VI= secundária inicial atrativa à fauna e ativadora da sucessão e VII= secundária tardia à sombra atrativa a fauna)

\begin{tabular}{lcccccc}
\hline Espécies & DZ & CR & FBN & Atrativa & Ativadora & Grupo \\
\hline Aiouea saligna & $\mathrm{X}$ & - & - & $\mathrm{x}$ & - & $\mathrm{I}$ \\
Alchornea triplinervia & $\mathrm{X}$ & $\mathrm{x}$ & - & $\mathrm{x}$ & $\mathrm{x}$ & $\mathrm{VI}$ \\
Alibertia edulis & $\mathrm{X}$ & - & - & $\mathrm{x}$ & - & $\mathrm{VII}$ \\
Aloysia virgata & - & $\mathrm{x}$ & - & - & $\mathrm{x}$ & $\mathrm{II}$ \\
Amaioua guianensis & $\mathrm{X}$ & - & - & $\mathrm{x}$ & - & $\mathrm{VII}$ \\
Annona acutiflora & $\mathrm{X}$ & $\mathrm{x}$ & - & $\mathrm{x}$ & $\mathrm{x}$ & $\mathrm{VI}$ \\
Aspidosperma cylindrocarpon & - & - & - & - & - & $\mathrm{IV}$ \\
Aspidosperma polyneuron & - & - & - & - & - & $\mathrm{VII}$ \\
Astronium concinnum & - & - & - & - & - & $\mathrm{VII}$ \\
Bauhinia longifolia & - & - & $\mathrm{x}$ & - & $\mathrm{x}$ & $\mathrm{II}$ \\
Blepharocalyx salicifolius & $\mathrm{X}$ & - & - & $\mathrm{x}$ & - & $\mathrm{IV}$ \\
Byrsonima crassifolia & $\mathrm{X}$ & - & - & $\mathrm{x}$ & - & $\mathrm{IV}$ \\
Cabralea canjerana & $\mathrm{X}$ & - & - & $\mathrm{x}$ & - & $\mathrm{VII}$ \\
Calyptranthes clusiifolia & $\mathrm{X}$ & - & - & $\mathrm{x}$ & - & $\mathrm{IV}$
\end{tabular}




\begin{tabular}{|c|c|c|c|c|c|c|}
\hline Espécies & DZ & $\mathbf{C R}$ & FBN & Atrativa & Ativadora & Grupo \\
\hline Calyptranthes concinna & $\mathrm{X}$ & - & - & $\mathrm{x}$ & - & $\bar{I}$ \\
\hline Calyptranthes lucida & $\mathrm{X}$ & - & - & $\mathrm{x}$ & - & VII \\
\hline Casearia arborea & $\mathrm{X}$ & - & - & $\mathrm{x}$ & - & IV \\
\hline Chomelia brasiliana & $\mathrm{X}$ & - & - & $\mathrm{x}$ & - & VII \\
\hline Cinnamomum erythropus & $\mathrm{X}$ & - & - & $\mathrm{x}$ & - & IV \\
\hline Citharexylum myrianthum & $\mathrm{X}$ & - & - & $\mathrm{x}$ & - & IV \\
\hline Copaifera langsdorffii & $\mathrm{X}$ & - & $\mathrm{x}$ & $\mathrm{x}$ & $\mathrm{x}$ & VI \\
\hline Cordia ecalyculata & $\mathrm{X}$ & - & - & $\mathrm{x}$ & - & IV \\
\hline Cordiera sessilis & $\mathrm{X}$ & - & - & $\mathrm{x}$ & - & I \\
\hline Coussarea hydrangeifolia & $\mathrm{X}$ & - & - & $\mathrm{x}$ & - & I \\
\hline Coutarea hexandra & - & - & - & - & - & IV \\
\hline Croton piptocalyx & - & $\mathrm{x}$ & - & - & $\mathrm{x}$ & II \\
\hline Cupania vernalis & $\mathrm{X}$ & - & - & $\mathrm{x}$ & - & IV \\
\hline Cybistax antisyphilitica & - & - & - & - & - & IV \\
\hline Dalbergia nigra & - & - & $\mathrm{x}$ & - & $\mathrm{x}$ & $\mathrm{V}$ \\
\hline Dalbergia villosa & - & - & $\mathrm{x}$ & - & $\mathrm{x}$ & $\mathrm{V}$ \\
\hline Diatenopteryx sorbifolia & - & - & - & - & - & I \\
\hline Dilodendron bipinnatum & $\mathrm{X}$ & - & - & $\mathrm{x}$ & - & I \\
\hline Diplotropis ferruginea & - & - & $\mathrm{x}$ & - & $\mathrm{x}$ & II \\
\hline Eremanthus erythropappus & - & $\mathrm{x}$ & - & - & $\mathrm{x}$ & III \\
\hline Esenbeckia febrifuga & - & - & - & - & - & IV \\
\hline Eugenia acutata & $\mathrm{X}$ & - & - & $\mathrm{x}$ & - & VII \\
\hline Eugenia brasiliensis & $\mathrm{X}$ & - & - & $\mathrm{x}$ & - & VII \\
\hline Eugenia florida & $\mathrm{X}$ & - & - & $\mathrm{x}$ & - & VII \\
\hline Eugenia francavilleana & $\mathrm{X}$ & - & - & $\mathrm{x}$ & - & VII \\
\hline Eugenia involucrata & $\mathrm{X}$ & - & - & $\mathrm{x}$ & - & IV \\
\hline Eugenia pyriformis & $X$ & - & - & $\mathrm{x}$ & - & VII \\
\hline Ficus gomelleira & $\mathrm{X}$ & - & - & $\mathrm{x}$ & - & IV \\
\hline Garcinia gardneriana & $\mathrm{X}$ & - & - & $\mathrm{x}$ & - & IV \\
\hline Guatteria sellowiana & $\mathrm{X}$ & - & - & $\mathrm{x}$ & - & IV \\
\hline Hortia brasiliana & $\mathrm{X}$ & - & - & $\mathrm{x}$ & - & VII \\
\hline Hymenolobium heringerianum & - & - & $\mathrm{x}$ & - & $\mathrm{x}$ & $\mathrm{V}$ \\
\hline Hyptidendron asperrimum & $\mathrm{X}$ & - & - & $\mathrm{x}$ & - & IV \\
\hline Ilex affinis & $\mathrm{X}$ & - & - & $\mathrm{x}$ & - & IV \\
\hline Ilex theezans & $\mathrm{X}$ & $\mathrm{x}$ & - & $\mathrm{x}$ & $\mathrm{x}$ & VI \\
\hline Kielmeyera lathrophyton & - & - & - & - & - & I \\
\hline Mabea fistulifera & - & $\mathrm{x}$ & - & - & $\mathrm{x}$ & II \\
\hline Machaerium nyctitans & - & - & $\mathrm{x}$ & - & $\mathrm{x}$ & V \\
\hline Machaerium stipitatum & - & - & $\mathrm{x}$ & - & $\mathrm{x}$ & $\mathrm{V}$ \\
\hline Machaerium villosum & - & $\mathrm{x}$ & $\mathrm{x}$ & - & $\mathrm{x}$ & V \\
\hline Manihot brachyloba & - & $\mathrm{x}$ & - & - & $\mathrm{x}$ & II \\
\hline Maprounea guianensis & - & - & - & - & - & IV \\
\hline Matayba elaeagnoides & $\mathrm{X}$ & - & - & $\mathrm{x}$ & - & I \\
\hline
\end{tabular}




\begin{tabular}{|c|c|c|c|c|c|c|}
\hline Espécies & $\mathbf{D Z}$ & $\mathbf{C R}$ & FBN & Atrativa & Ativadora & Grupo \\
\hline Maytenus boaria & $\mathrm{X}$ & - & - & $\mathrm{x}$ & - & VII \\
\hline Miconia brunnea & $\mathrm{X}$ & - & - & $\mathrm{x}$ & - & VII \\
\hline Miconia cinnamomifolia & $\mathrm{X}$ & - & - & $\mathrm{x}$ & - & I \\
\hline Myracrodruon urundeuva & - & - & - & - & $\mathrm{x}$ & II \\
\hline Myrcia pulchra & $\mathrm{X}$ & $\mathrm{x}$ & - & $\mathrm{x}$ & $\mathrm{x}$ & VII \\
\hline Myrcia retorta & $\mathrm{X}$ & - & - & $\mathrm{x}$ & - & I \\
\hline Myrcia splendens & $\mathrm{X}$ & - & - & $\mathrm{x}$ & - & IV \\
\hline Myrsine gardneriana & $\mathrm{X}$ & - & - & $\mathrm{x}$ & - & IV \\
\hline Myrsine guianensis & $\mathrm{X}$ & $\mathrm{x}$ & - & $\mathrm{x}$ & $\mathrm{x}$ & III \\
\hline Nectandra cissiflora & $\mathrm{X}$ & - & - & $\mathrm{x}$ & - & VII \\
\hline Nectandra lanceolata & $\mathrm{X}$ & - & - & $\mathrm{x}$ & - & VII \\
\hline Ocotea pulchella & $\mathrm{X}$ & - & - & $\mathrm{x}$ & - & IV \\
\hline Picrasma crenata & $\mathrm{X}$ & - & - & $\mathrm{x}$ & - & IV \\
\hline Pimenta pseudocaryophyllus & $\mathrm{X}$ & $\mathrm{x}$ & - & $\mathrm{x}$ & $\mathrm{x}$ & III \\
\hline Piptadenia gonoacantha & - & $\mathrm{x}$ & $\mathrm{x}$ & - & $\mathrm{x}$ & II \\
\hline Plinia grandifolia & $\mathrm{X}$ & - & - & $\mathrm{x}$ & - & IV \\
\hline Pouteria ambelaniifolia & $\mathrm{X}$ & - & - & $\mathrm{x}$ & - & VII \\
\hline Pouteria caimito & $\mathrm{X}$ & - & - & $\mathrm{x}$ & - & IV \\
\hline Protium heptaphyllum & $\mathrm{X}$ & - & - & $\mathrm{x}$ & - & I \\
\hline Psychotria vellosiana & $\mathrm{X}$ & - & - & $\mathrm{x}$ & - & IV \\
\hline Rudgea gardenioides & $\mathrm{X}$ & - & - & $\mathrm{x}$ & - & VII \\
\hline Siparuna guianensis & - & $\mathrm{x}$ & - & - & $\mathrm{x}$ & $\mathrm{V}$ \\
\hline Siphoneugena densiflora & $\mathrm{X}$ & - & - & $\mathrm{x}$ & - & VII \\
\hline Sorocea bonplandii & $\mathrm{X}$ & - & - & $\mathrm{x}$ & - & IV \\
\hline Swartzia myrtifolia & $\mathrm{X}$ & - & $\mathrm{x}$ & $\mathrm{x}$ & $\mathrm{x}$ & $\mathrm{V}$ \\
\hline Tachigali rugosa & - & - & $\mathrm{x}$ & - & $\mathrm{x}$ & $\mathrm{V}$ \\
\hline Tapirira guianensis & $\mathrm{X}$ & $\mathrm{x}$ & - & $\mathrm{x}$ & $\mathrm{x}$ & VI \\
\hline Tapirira obtusa & $\mathrm{X}$ & $\mathrm{x}$ & - & $\mathrm{x}$ & $\mathrm{x}$ & VI \\
\hline Toulicia laevigata & - & - & - & - & - & IV \\
\hline Trichilia hirta & $\mathrm{X}$ & - & - & $\mathrm{x}$ & - & IV \\
\hline Vismia brasiliensis & $X$ & - & - & $\mathrm{x}$ & - & IV \\
\hline Xylopia brasiliensis & $\mathrm{X}$ & $\mathrm{x}$ & - & $\mathrm{x}$ & $\mathrm{x}$ & VII \\
\hline Xylopia sericea & $\mathrm{X}$ & $\mathrm{x}$ & - & $\mathrm{x}$ & $\mathrm{x}$ & VII \\
\hline $\begin{array}{l}\text { DISCUSSÃO } \\
\text { A riqueza no compartimento adulto } \\
\text { considerada baixa quando comparada com o } \\
\text { trabalhos que utilizaram pontos quadrante } \\
\text { apresentados na literatura (LOPES et al., 200 } \\
\text { CATHARINO et al., 2006). A diferença na riquez } \\
\text { entre os compartimentos pode ter ocorrido devido } \\
\text { provavelmente, que esta área não tenha atingindo } \\
\text { clímax dinâmico, e assim apresentando diferença d } \\
\text { espécies entres os mesmos. } \\
\text { O teste de Qui-quadrado para as guildas d } \\
\text { regeneração também evidencia que a floresta poss }\end{array}$ & & \multicolumn{5}{|c|}{$\begin{array}{l}\text { fase intermediária de sucessão e não houve } \\
\text { diferença significativa entre os compartimentos } \\
\text { estudados. } \\
\text { A riqueza variou de forma considerável } \\
\text { entre os compartimentos. Entretanto, a } \\
\text { complexidade dos compartimentos permaneceu } \\
\text { próxima, como pode verificar pelos índices de } \\
\text { diversidade. Mesmo apresentando valores próximos } \\
\text { no índice de diversidade de Shannon, os } \\
\text { compartimentos adulto e juvenil apresentam } \\
\text { estruturas diferentes, a riqueza foi maior no } \\
\text { compartimento juvenil e o compartimento adulto } \\
\text { apresenta maior equabilidade. }\end{array}$} \\
\hline
\end{tabular}


A composição florística foi comparada por diferentes métodos de amostragem em Floresta Estacional Semidecidual (FES) Montana: Dias-Neto et al. (2009), Vale et al. (2009); Meira-Neto e Martins (2002), Machado (2004) e Pereira et al. (2006), os quais utilizaram o método de parcelas permanentes

(MUELLER-DOMBOIS; ELLENBERG, 1974). Pelo método de pontos quadrantes em regiões de Minas Gerais, comparouse com os trabalhos de Lopes et al. (2009) e o de Santos (2009). Entre este e os trabalhos supracitados percebe-se um padrão comum quanto à riqueza no nível de família, sendo Myrtaceae, Fabaceae, Lauraceae Melastomataceae e Rubiaceae repetidamente as famílias com maior número de espécies, sugerindo ampla distribuição dessas em áreas de floresta Montana em Minas Gerais. Pereira et al. (2006) afirmam que esse padrão de riqueza no nível de famílias é característico das florestas situadas acima de $1.000 \mathrm{~m}$ de altitude no Sudeste do Brasil. Oliveira-Filho e Fontes (2000) observaram um perfil florístico semelhante ao encontrado neste trabalho, estudando florestas de altitude do sudeste do Brasil, sendo as famílias com maior número de espécies foram Myrtaceae, Fabaceae, Lauraceae, Melastomataceae e Rubiaceae.

A densidade de indivíduos jovens foi muito superior quando comparado aos trabalhos de Gonzaga et al., $2008(2386,66)$ em Tiradentes, MG e Martins et al., 2002 (833) em Viçosa, MG. Em relação à riqueza, Marangon et al. (2008), estudando a regeneração natural por método de parcelas em uma Floresta Estacional Semidecídua na região da Zona da Mata mineira, encontrou 91 espécies.
As altas frequências de espécies que têm suas fontes de propágulos dispersadas por animais (67\%) são comumente encontradas nas florestas em ambientes tropicais, sendo importantes recursos para a sobrevivência de animais (KARR et al., 1990). Esses resultados indicam que a comunidade apresenta características semelhantes às demais florestas estacionais semideciduais estudadas (DIAS-NETO et al., 2009; VALE et al., 2009; PEREIRA et al.; 2006).

A caracterização das espécies em grupos funcionais, de acordo com as características ecológicas e silviculturais, é importante para a determinação das espécies que possui características potencialmente facilitadoras de sucessão. Estas características são fundamentais em áreas degradadas pela mineração, pois estes locais encontram-se com solos em condições físicas, químicas e ou biológicas alteradas (PEREIRA et al., 2010).

\section{CONCLUSÕES}

A avaliação da flora arbustiva-arbórea dos compartimentos foi essencial para o conhecimento das possíveis espécies a serem utilizadas na revegetação de áreas degradadas por mineração de ferro em ecossistemas congêneres.

A partir das características ecológicas das espécies é possível definir grupos funcionais que poderão ser utilizados na recuperação de áreas degradadas.

\begin{abstract}
The objective of this study was to evaluate the tree flora in a fragment of semi deciduous forest in the region of the Espinhaço, with aim to propose species for restoration of areas degraded by mining exploitation of iron in similar ecosystems. The inventory floristic and structural of vegetation of shrubs and trees was conducted in September 2007, by the quadrants-point method according to the method of minimum distances, whose inclusion criterion of the stem above the height $1.3 \mathrm{~m}$. The individual were stratified into adults $(D B H \geq 5.0 \mathrm{~cm})$ and juvenile $(D B H<5.0 \mathrm{~cm})$. We sampled 90 points, which were identified 94 species, 70 genera and 32 families. For the diversity index of Shannon, values presented for each layer were 3.96 nats.ind $^{-1}$ and 3.94 nats.ind $^{-1}$, and equability of Pielou were 0.896 and 0.938 , respectively for layer juvenile and adult. Families that stood out by the number of species were Myrtaceae (17), Fabaceae (12), Rubiaceae (8), Euphrobiaceae (6), Sapindaceae (5) and Lauraceae (5). For the recovery area were selected seven groups of species that will be used for recuperation of degraded areas. The characteristics of the species were sufficient for the definition of functional groups that can be used in recuperation of degraded areas.
\end{abstract}

KEYWORDS: Environmental restoration. Planting models. Functional groups and ecological succession

\title{
REFERÊNCIAS
}

APG III - An update of the Angiosperm Phylogeny Group classification for the orders and families of flowering plants. Botanical Journal of the Linnean Society, Londres, v. 161, p. 105-121. With 1 figure, 2009. 
CARVALHO, P. E. R. 2003. Espécies Arbóreas Brasileiras. Vol. 1. Colombo: Embrapa Informação Tecnológica. Embrapa Floresta.

CARVALHO, P. E. R. 2006. Espécies Arbóreas Brasileiras. Vol. 2. Colombo: Embrapa Informação Tecnológica. Embrapa Floresta.

CARVALHO, P. E. R. 2008. Espécies Arbóreas Brasileiras. Vol. 3. Colombo: Embrapa Informação Tecnológica. Embrapa Floresta.

CATHARINO, E.L.M. BERNACCI, L. C.; FRANCO, G. A. D. C.; DURIGAN, G.; METZGER, J. P. Aspectos da composição e diversidade do componente arbóreo das florestas da Reserva Florestal do Morro Grande, Cotia, SP. Biota Neotropica, São Paulo, v. 6, n. 2, p. 1-28, 2006.

COTTAM, G.; CURTIS, J. T. The use of distance measure in phytosociological sampling. Ecology, Washington, v. 37, p. 451-461, 1956.

DIAS-NETO, O. C.; SCHIAVINI, I.; LOPES, S. F.; et al. Estrutura fitossociológica e grupos ecológicos em fragmento de floresta estacional semidecidual, Uberaba, Minas Gerais, Brasil. Rodriguésia, Rio de Janeiro, v. 60, n. 4, p. 1087-1100, 2009.

DURIGAN, G.; SIQUEIRA, M. F.; FRANCO, G. A. D. C.; CONTIERI, W.A. Flora arbustiva-arbórea do médio Paranapanema: base para a restauração dos ecossistemas naturais. In: VILAS BOAS, O.; DURIGAN, G. (Orgs.) Pesquisas em conservação e recuperação ambiental no oeste paulista: resultados da cooperação Brasil/Japão. São Paulo: Secretaria do Meio Ambiente/IF/JICA, 2004. p.199-239.

GANDOLFI, S.; LEITÃO FILHO, H. F.; BEZERRA, C. L. F. Levantamento florístico e caráter sucessional das espécies arbustivo-arbóreas de uma floresta mesófila semidecídua no município de Guarulhos, SP. Revista Brasileira de Biologia, Rio de Janeiro, v. 55, n. 4, p. 753-767, 1995.

GONTIJO, B. M. Uma geografia para a Cadeia do Espinhaço. Megadiversidade, v. 4, p. 7-15, 2008.

GONZAGA, A. P. D.; OLIVEIRA-FILHO, A. T.; MACHADO, E. L. M.; et al. Diagnóstico florísticoestrutural do componente arbóreo da floresta da Serra de São José, Tiradentes, MG, Brasil. Acta Botanica Brasilica, São Paulo, v. 22, n. 2, p. 505-520, 2008. http://dx.doi.org/10.1590/S0102-33062008000200018

HARLEY, R. M. Introduction in Flora of the Pico das Almas, Chapada Diamantina, Brazil. In: StANNARD, B. L.; HARVEY, Y. B.; HARLEY, R. M. Royal Botanic Gardens, p. 43-79, 1995.

IBGE. Instituto Brasileiro de Geografia e Estatística. Mapa de vegetação do Brasil. IBGE, Rio de Janeiro. 1993.

KARR, J. R.1990. The avifauna of Barro Colorado Island and the Pipeline Road, Panama. In: GENTRY, A. H. (ed.) Four neotropical rainforests: 183-198. New Haven, Connecticut, USA; Yale University Press.

LANA, C. E.; ALVES, J. M. P.; CASTRO, P. T. A. Análise morfométrica da bacia do Rio do Tanque, MG Brasil. Rev. Esc. Minas, Ouro Preto, v. 54, n. 2, p. 121-126, 2001.

LOPES, R. M. F.; FRANÇA, G. S.; SILVA, F. R. G.; et al. Estrurura do componente arbóreo de Floresta Estacional Semidecidual montana secundária no Alto Rio Doce, Minas Gerais, Brasil. Rodriguésia, Rio de Janeiro, v. 60, n. 4, p. 1037-1053, 2009.

LOPES, S. F.; OLIVEIRA, A. P.; DIAS-NETO, O. C.; et al. Estrutura e Grupos Ecológicos em uma Floresta Estacional Semidecidual em Uberlândia, MG. In: SIMPÓSIO INTERNACIONAL DE SAVANNAS TROPICAIS, 2., 2008, Brasília - DF. Anais... Brasilia: 2008. 
MACHADO, E. L. M.; OLIVEIRA-FILHO, A. T.; CARVALHO, W. A. C.; et al. Análise comparativa da estrutura e flora do compartimento Arbóreo-arbustivo de um remanescente florestal na fazenda Beira lago, Lavras, MG. Revista Árvore, Viçosa, v. 28, n. 4, p. 499-516, 2004.

MARANGON, L. C.; SOARES, J. J. ; FELICIANO, A. L. P.; BRANDÃO, C. F. L. S. Regeneração natural em um fragmento de floresta estacional semidecidual em Viçosa, Minas Gerais. Revista Árvore, Viçosa, v. 32, p. 183-191, 2008.

MARTINS, Fernando Roberto. O Método dos quadrantes e a fitossociologia de uma floresta residual no interior do Estado de São Paulo: Parque Estadual de Vassununga. 1979. 239 p. Tese (Doutorado em Botânica) - Curso de Pós-graduação em Botânica, Instituto de Biociências da Universidade de São Paulo, São Paulo, 1979.

MARTINS, S. V.; RIBEIRO, G. A.; SILVA-JUNIOR, W. M.; NAPPO, M. E. Regeneração pós-fogo em um fragmento de floresta estacional semidecidual no município de Viçosa, MG. Revista Ciência Florestal, Santa Maria, v. 12, n. 1, 2002.

MEIRA-NETO, J. A. A.; MARTINS, F. R. Composição florística de uma Floresta Estacional Semidecidual Montana no Município de Viçosa-MG. Revista Árvore, Viçosa, v. 26, n. 4, p. 437-446, 2002.

MUELLER-DOMBOIS; D, ELLENBERG, H. Aims and methods of vegetation ecology. New York, John Wiley e Sons, 547p. 1974.

MYERS, N.; MITTERMEIER, R. A.; MITTERMEIER, C. G.; et al. Biodiversity hotspots for conservation priorities. Nature, Londres, n. 403, p. 853-858. 2000.

OLIVEIRA-FILHO, A. T.; FONTES, M. A. L. Patterns of Floristic Differentiation among Atlantic Forests in Southeastern Brazil and the Influence of Climate. Biotropica, Washington, v. 32, p. 793-810, 2000.

http://dx.doi.org/10.1111/j.1744-7429.2000.tb00619.x

PAULA, A.; SILVA, A. F.; MARCO-JÚNIOR, P.; et al. Sucessão ecológica da vegetação arbórea em uma Floresta Estacional Semidecidual, Viçosa, MG, Brasil. Acta Botânica Brasilica, São Paulo, v. 18, n. 3, p. 407423. 2004. http://dx.doi.org/10.1590/S0102-33062004000300002

PEREIRA, I. M.; BOTELHO, S. A.; VAN DER BERG, E.; et al. Caracterização ecológica de espécies arbóreas ocorrentes em ambientes de mata ciliar, como subsídio à recomposição de áreas alteradas nas cabeceiras do rio grande, Minas Gerais, Brasil. Revista Ciência Florestal, Santa Maria, v. 20, n.2, p. 235-253, 2010.

PEREIRA, I. M.; OLIVEIRA-FILHO, A. T.; BOTELHO, S. A.; et al. Composição florística do compartimento arbóreo de cinco remanescentes florestais do maciço do Itatiaia, Minas Gerais e Rio de Janeiro. Rodriguésia, Rio de Janeiro, v. 57, p. 103-126, 2006.

PEREIRA, I. M. Estudo da vegetação remanescente como subsídio à recomposição de áreas ciliares nas cabeceiras do Rio Grande, Minas Gerais. 2006.261 f. Tese (Doutorado em Engenharia Florestal) - Curso de Pós-graduação em Engenharia Florestal, área de concentração Manejo Ambiental, Universidade Federal de Lavras, Lavras, 2006.

RESSEL, K., GUILHERME, F. A. G., SCHIAVINI, I.; OLIVEIRA, P. E. Ecologia morfofuncional de plântulas de espécies arbóreas da Estação Ecológica do Panga, Uberlândia, Minas Gerais. Revista Brasileira de Botânica, São Paulo, v. 27, n. 2, p. 311-323, 2004.

SANTOS, M. F. Análise florística em Floresta Estacional Semidecidual na encosta leste da serra do cipó, MG. 2009. Dissertação (Mestrado em Botânica) - Curso de Pós-graduação em Botânica, Instituto de Biociência da Universidade de São Paulo, São Paulo, 2009. 
SILVA, N. R. S. Florística e estrutura horizontal de uma Floresta Estacional Semidecidual Montana mata do Juquinha de Paula, Viçosa, MG. 2002. 83f. Dissertação (Mestrado em Ciências Florestais) - Curso de Pós-graduação em Ciências Florestais, Universidade Federal de Viçosa, Viçosa, 2002.

SOUZA, A. L.; SCHETTINO, S.; JESUS, R. M.; VALE, A. B. Dinâmica da regeneração natural em uma floresta ombrófila densa secundária, após corte de cipós, reserva natural da Companhia Vale do Rio Doce S.A., Estado do Espírito Santo, Brasil. Revista Árvore, Viçosa, v. 26, n. 4, p. 411-419. 2002.

TAKAHASHI, A.; FIANA, B. G. Síndromes de dispersão de sementes de uma área do Morro do Paxixi, Aquidauana, MS. In: SIMPÓSIO SOBRE RECURSOS NATURAIS E SÓCIO-ECONÔMICOS DO PANTANAL, 4., Corumbá, 2004.Anais... Corumbá: 2004.

TEIXEIRA, W. A.; LEMOS FILHO, J. P. Fatores edáficos e a olonização de espécies lenhosas em uma cava de mineração de ferro em Itabirito, Minas Gerais. Revista Árvore, Viçosa, v. 26, p. 25-33. 2002.

VALE, V.S.; SCHIAVINI, I. ; LOPES, S. F.; et al. Composição florística e estrutura do componente arbóreo em um remanescente primário de floresta estacional semidecidual em Araguari, Minas Gerais, Brasil.

Hoehnea, São Paulo, v. 36, n. 3, p. 417-429, 3 tab., 2009.

VAN DER PIJL, L. Principles of dispersal in higher plants. 3rd ed. Springer-Verlarg, Berlin. 1982. http://dx.doi.org/10.1007/978-3-642-87925-8

VELOSO, H. P.; RANGEL FILHO, A. L. R.; LIMA, J. C. A. 1991. Classificação da vegetação brasileira adaptada a um sistema universal. Instituto Brasileiro de Geografia e Estatística, Rio de Janeiro.

VIEIRA, F., G. B. SANTOS; C. B. M. ALVES. A ictiofauna do Parque Nacional da Serra do Cipó (Minas Gerais, Brasil) e áreas adjacentes. Lundiana, Belo Horizonte, v. 6, p. 77-87, 2005.

YAMAMOTO, L. F., KINOSHITA, L. S.; MARTINS, F. R.. Síndromes de polinização e de dispersão em fragmentos da Floresta Estacional Semidecídua Montana, SP, Brasil. Acta Botânica Brasilica, São Paulo, v. 21, n. 3, p. 553-573, 2007. http://dx.doi.org/10.1590/S0102-33062007000300005

ZAR, J. H. 1996. Biostatistical analysis, 3.ed. Prentice Hall, New Jersey. 